\title{
EL EDUCANDO COMO SUJETO Y EL LUGAR DEL JUEGO EN EL DEBATE EDUCATIUO DE FInALES DEL SIGLO XIX En NORTEAmÉRICA
} The Learner as Subject and the Place of Play and Games in the
Educational Debate in Morth-America at the end of $19^{\text {th }}$ Century

ROSA BRUNO-JOFRÉ Queen's University

GONZALO JOVER

Universidad Complutense

El artículo examina la construcción de la idea de sujeto y las nociones correspondientes del juego en tres de los credos pedagógicos publicados en The School Journal (Nueva York y Chicago) entre 1896 y 1897. Los tres credos son los del estadounidense William Torrey Harris (18351909), humanista hegeliano, comisario del Bureau of Education de EE UU; James L. Hughes (1846-1935), canadiense, inspector escolar en Toronto, promotor de las ideas de Froebel; y Edward W. Scripture (1864-1945), profesor de Psicología de Yale University, especialista en psicología experimental. El marco teórico del análisis lo proporciona la diferenciación de Jerrold Seigel de tres dimensiones de la mismidad: material o corporal, relacional y reflexiva.

El trabajo mantiene que en Harris predomina una visión del educando fuertemente relacional, enfocada a la integración del individuo en el todo social. Hughes concibe la autoactividad como elemento mediador entre espontaneidad y control, teniendo como marco de referencia una noción de libertad que no puede ser entendida sin conciencia de lo que significa la restricción. Scripture representa el cambio de paradigma que estaba teniendo lugar. Su visión del yo y del educando como sujeto está influida por las ciencias naturales y el empirismo emergente, en línea con el pensamiento positivista. Los tres autores entienden el juego y el juego regulado como un medio de desarrollo de un sujeto autocontrolado en respuesta a una intencionalidad externa, incluso cuando el debate se mueve a la discusión entre idealismo y realismo, este último expresado por Scripture, con lo que el juego, y no solo el regulado, termina por perder su significado como actividad estrictamente lúdica.

Palabras clave: Educación en el siglo XIX, Educando, Sujeto, Yo, Juego, Juego regulado. 


\section{Introducción}

Durante 1896 y 1897, The School Journal, revista publicada en Nueva York y Chicago, pidió a dieciséis líderes en educación, protagonistas del debate del momento, que escribieran sus credos pedagógicos. Ossian Lang, editor ejecutivo del Journal, solicitó a los autores «declaraciones breves pero comprensivas de los ideales educativos y planes en cuya implementación basarían sus esperanzas para el futuro de la civilización americana» (Lang, 1898: V). La intención, como se señala en una nota de la revista a uno de los credos, era familiarizar a los maestros con ideas claras acerca de los fines de la educación (The School Journal, 1896: 662). El credo de John Dewey, publicado por primera vez en esta colección, se convertiría en el más conocido e influyente.

Los credos reflejan complejas configuraciones en las que interactúan el evolucionismo de Darwin y de Spencer (que aportó al debate visiones neolamarkianas), las ideas relacionadas con el método científico, el evangelio social protestante, el reconstruccionismo social, las teorías de la centralidad del niño/a en el proceso educativo, nuevos desarrollos en psicología que llevarían a la americanización de la psicología experimental, en particular de las ideas de Wundt, y al conductismo (Tröhler, 2011). La influencia de la tradición pedagógica europea y el neohumanismo alemán en Norteamérica es bien conocida, si bien se debaten las características que adoptó la recepción y su perdurabilidad ${ }^{1}$. Pestalozzi, Froebel, Diesterweg, Herbart, Spencer, la filosofía neohegeliana, y la psicología experimental alemana están presentes de diferentes maneras en los credos. El movimiento transnacional de ideas educativas se plasmaría pronto en la formación de redes y sociedades y en la organización de conferencias internacionales sobre la nueva educación ${ }^{2}$.

La publicación de los credos coincide con un periodo intenso de la historia de Estados Unidos, embarcados en la reconstrucción nacional tras la guerra civil, los problemas raciales que siguieron a la abolición de la esclavitud, la cuestión de las poblaciones indígenas, los movimientos a favor del sufragio de la mujer, las tensiones sociales provocadas por el rápido proceso de industrialización, la afluencia masiva de inmigrantes, que muchos vivían como una amenaza a la identidad nacional y religiosa, y el surgimiento de una nueva elite social basada en las grandes corporaciones. A finales del siglo XIX, despuntaba ya el predominio que Estados Unidos llegaría a tener en el panorama mundial en el siglo siguiente. Un predominio sustentado sobre diversas fracturas sociales que para muchos auguraba un futuro sombrío. «En la década de 1890 — cuando se escriben los credos- solo los que estaban a cubierto o los optimistas podían pensar que la sociedad estadounidense no iba a necesitar algún tipo de transformación social radical en el siglo siguiente, aunque no había acuerdo sobre adónde podría llevarles ese nuevo rumbo» (Jenkins, 2010: 241).

Los credos plasman esta realidad, aunque en cierto sentido también la ocultan. Su estilo es el de una perspectiva universalista que en ocasiones resulta alejada de las circunstancias socioeconómicas y de las exclusiones sociales. En ellos no están todas las voces; la colección es selectiva. No hay entre los autores ninguna mujer, a pesar de que en el panorama pedagógico del momento destacaban nombres como los de Susan Blow, vinculada al movimiento de los Kindergarten. También falta, por ejemplo, Booker T. Washington, el pedagogo afroamericano más influyente de esa época.

En otros trabajos, hemos estudiando el contexto y contenido de los credos en intersección especialmente con la cuestión religiosa (BrunoJofré y Jover, 2009, 2011). Aquí nos vamos a centrar en tres de ellos. A partir de estos textos, abordaremos un momento específico de la construcción histórica del educando como sujeto que fundamenta las nociones de autonomía y responsabilidad, centrales a la concepción moderna de la educación, y la concepción 
El educando como sujeto y el lugar del juego en el debate educativo de finales del siglo XIX en Norteamérica

del juego que emana en cada uno de ellos de su noción del sujeto.

El primero de los tres textos es el credo de William Torrey Harris (1835-1909), quien adopta las ideas de Hegel a la luz de la transición intelectual, social y política, pero no asume educativamente esa realidad en su teoría, sino que mantiene una visión del mundo ya ajena a la realidad histórica. Así defiende en la práctica, entre otras cosas, un currículo humanista con alguna desconfianza hacia el énfasis en la ciencias naturales (Kliebard, 1986: 17). El segundo texto es el de James L. Hughes (18461935), canadiense, inspector de escuelas en la ciudad de Toronto y Grand Master de la Logia Orange del oeste de Ontario. Este articula nociones que están en la base de una mentalidad protestante, en especial del emergente evangelio social que toma forma a fines del siglo XIX, con una visión evolucionista del individuo y la sociedad, la noción de autoactividad (self activity) y el concepto de unidad que revela la lectura de Froebel ${ }^{3}$. Por último, el tercer texto considerado es el credo de Edward W. Scripture (1864-1945), profesor y director del laboratorio de Psicología de Yale University. Representa una dirección diferente. Su interés no estaba en conocer mejor el alma, sino la conducta humana y su variabilidad. Encarna lo que Daniel Tröhler define como la americanización de las ideas de Wundt, lo que da lugar a una psicología enfocada a medir las experiencias interiores, las cuales no se consideran, sin embargo, opuestas a las experiencias exteriores (Tröhler, 2011: 137).

El marco teórico del análisis está dado por un enfoque hermenéutico orientado hacia la manera en que los tres autores entendieron, interpretaron y visualizaron la concepción del educando y la idea del yo y la mismidad, con sus correspondientes conceptos morales. Para examinar la idea del yo y la mismidad y la noción de educando en las concepciones educativas expuestas en los credos de Harris, Hughes y Scripture, acudimos al trabajo de Jerrold
Seigel The Idea of the Self: Thought and Experience in Western Europe Since The Seventeenth Century (Seigel, 2005). Este ha señalado que en la cultura occidental las bases del yo y la mismidad se han explorado siguiendo tres dimensiones: la corporal o material, la relacional y la reflexiva (ibíd.: 5-6). La primera incluye los aspectos de nuestra naturaleza que nos hacen criaturas palpables, movidas por necesidades, urgencias e inclinaciones, y la conciencia que tenemos de todo esto. El movimiento hacia la centralidad del niño/a en el proceso educativo, la preocupación por sus inclinaciones, intereses y deseos son maneras de encarar la dimensión material, aunque esta no pueda separarse de las otras dimensiones. La forma de entender esta dimensión está muy relacionada con la concepción de la educación y del proceso educativo, así como con ideas acerca de la naturaleza del niño/a. La segunda, relacional, es una dimensión que tiene que ver con la interacción cultural y social, las conexiones, lo que nos da una identidad colectiva, valores compartidos, un idioma común. Esta dimensión es importante aquí porque el yo toma forma en el contexto de las relaciones con la sociedad, los valores, lo que es posible o permitido, y la escuela representa un microcosmos de ese contexto. La tercera dimensión, la de la reflexividad (reflectivity), deriva, según Seigel, de la capacidad del ser humano de observar el mundo y observarse a sí mismo de manera activa, incluyendo su cuerpo; le son inherentes la intención y el propósito, lo que la diferencia de la respuesta no intencional o refleja (reflexivity) (ibíd.: 12-13).

La forma en la que se entienden estas tres dimensiones y sus relaciones dependen de las posiciones filosóficas que se adopten. En nuestro caso, estas dimensiones sirven como instrumento para examinar la noción de sí mismo que permea el perfil del educando en los credos de Harris, Hughes y Scripture. La noción de sí mismo y mismidad está presente en varios credos, y a menudo estos términos se intercambian con los de individuo e individualidad. Como veremos, Harris, por 
ejemplo, entiende la educación como una condición esencial de la actividad de uno mismo (self); Hughes cree que la mismidad (selfhood) del niño/a es el elemento divino dentro de él/ella. Scripture se aleja de cualquier «metafísica mística» pero busca desarrollar un yo autocontrolado. Los autores representan corrientes de pensamiento y tradiciones filosóficas que se cruzan en el cambio de siglo, adaptadas al contexto. El humanismo racionalista de un Harris hegeliano, el froebelianismo místico de Hughes y el conductismo de Scripture proveen un marco de referencia contrastante de una época en crisis, y revelan la centralidad de las ideas del individuo y la mismidad en las concepciones modernas de la educación en la Norteamérica de finales del siglo XIX.

\section{Sujeto, juego y orden social: donde lo nuevo y lo viejo se separan, sintetizan en nuevas configuraciones o coexisten}

\section{El humanismo de William Torrey Harris}

Harris era una figura reconocida por su labor administrativa y su presencia intelectual en Estados Unidos. Había sido superintendente de Escuelas Públicas en St. Louis (1868-1880) y cuando se publicó el credo ocupaba el cargo de comisario del Bureau of Education (1889-1906). Fue uno de los impulsores del kindergarten froebeliano en los Estados Unidos ${ }^{4}$ y su obra pedagógica revela cierta influencia, entre otros, de Herbart, por ejemplo en el uso de la noción de apercepción, concepto herbatiano que se refiere a la asociación de nuevas ideas a las antiguas ideas de las que ya dispone el sujeto. Pero, fundamentalmente, Harris era un neohegeliano reconocido que jugó un papel central en el movimiento filosófico de St. Louis, liderado por Henry Conrad Brokmeyer; juntos fundaron el Journal of Speculative Philosophy. Trabajó durante varios años en la lógica de Hegel, a la que dedicó el libro Hegel's Logic: A Book on the
Genesis of the Categories of the min. A Critical Exposition ${ }^{5}$.

Lawrence Cremin interpreta la fascinación de Harris por la filosofía hegeliana, desde el lugar de transición que, a su juicio, este jugó en la historia de la educación americana:

«Harris, junto con muchos de sus compatriotas de mediados del siglo XIX, estaba sufriendo una profunda crisis religiosa [...]. Para Harris ni el naturalismo ni el misticismo resultaban suficientes. Sin embargo, en las doctrinas hegelianas de la dialéctica racionalista, de la verdad absoluta y de la autoactividad, encontró una forma - aunque tortuosa - de reconciliar las creencias cristianas de su niñez con los métodos y descubrimientos de la ciencia. En términos más generales, Harris utilizó a Hegel para confirmar lo que vale la pena conservar en una sociedad impregnada por el cambio. Este le permitió aceptar una nueva América sin repudiar la vieja. En el racionalismo de Hegel, Harris encontró la religión» (Cremin, 1964: 16).

Según esta tesis de Cremin, la dialéctica de Hegel proporciona a Harris los instrumentos para unir el mundo viejo, en el que se creó la escuela común, el mundo de su fe, y el mundo nuevo (conflictivo con una realidad social cruda); el idealismo y la teología individualista de salvación, por un lado, y el empuje científico (con sus varias dimensiones) y el progresismo social y educativo, por otro. Sin embargo, a la luz de la influencia hegeliana sobre otros autores, incluyendo Dewey, podríamos argumentar que Harris no pudo responder teóricamente a la necesidad de repensar la educación en relación con su papel en la democracia y con la crisis social generada por la revolución industrial, así como con la revolución científica.

El credo, escrito en un momento tardío de su carrera, intenta proteger el idealismo que siente amenazado y trata de conciliarlo con ideas que van adoptando nuevos significados. La condición para esta reconciliación es entender 
El educando como sujeto y el lugar del juego en el debate educativo de finales del siglo XIX en Norteamérica

el progreso individual como participación en el progreso colectivo expresado en la civilización y las instituciones. Harris abre el credo con una definición de educación entendida como «el proceso por el cual el individuo es elevado al nivel de la especie» (Harris, 1897: 813). Explica esta definición acudiendo a la noción de experiencia: «La educación da al individuo la sabiduría derivada de la experiencia de la raza» (ibíd.). Esta se refiere, en primer lugar, a la experiencia por la que la humanidad ha aprendido a conocer y dominar la naturaleza. Pero, la principal lección educativa es la que deriva de la experiencia de la naturaleza humana, manifestada en las costumbres y, sobre todo, en la evolución de las instituciones: la familia, la sociedad civil, el Estado, la Iglesia.

De la prioridad educativa de la experiencia, expresada en la sabiduría humana, sobre la naturaleza, derivan dos consecuencias. La primera es que el niño no puede entenderse como pura naturaleza individual. Harris considera la propuesta roussoniana de vuelta a la naturaleza «la herejía mas grande en la doctrina educativa» (ibíd.). Pero es igualmente crítico con quienes, basándose en el principio de la prioridad de la experiencia acumulada, desprecian las peculiaridades individuales $y$, sobre todo, restan valor a la autoactividad del niño y sus intereses, contentándose con que este obedezca las leyes del Estado, a sus padres y a sus mayores, tal como, afirma, sucede en China, en algunas monarquías europeas y, durante mucho tiempo, en las propias escuelas americanas.

La mismidad del educando en el credo de Harris se forma a través de la autoactividad del yo (self), donde puede verse la influencia de la pedagogía froebeliana reconstituida en los Estados Unidos. La educación, escribe Harris en el credo, tiene como condición esencial la actividad del yo, mientras que su fin es la elevación del individuo en la especie (ibíd.: 814). Es decir, destaca la dimensión relacional del niño/a como sujeto, ya que el sentido de su actividad se refuerza por la autoactividad de las instituciones y el todo social.

El detenimiento del desarrollo del niño y de su yo es motivo de preocupación. Se apoya en el spenceriano John Fiske para afirmar que la principal contribución de la teoría de la evolución a la pedagogía es hacernos ver cómo, en comparación con los animales, existe en el ser humano un largo periodo de infancia desvalida, que es requerido para el desarrollo de la adaptación a las costumbres y producciones culturales del entorno social. La insistencia a lo largo de cualquier etapa de este periodo de desarrollo sobre hábitos o formas de actividad correspondientes a etapas anteriores, paraliza o dificulta «el crecimiento del niño/a hacia formas más altas y más civilizadas de la actividad del alma» (ibíd.).

En el credo de Harris, en suma, autoactividad no significa tanto desarrollo libre de las capacidades individuales, como autoconciencia mediante la participación en el proyecto colectivo de la raza. En el juego dialéctico que defiende entre espontaneidad y prescripción, su pedagogía termina imponiendo el ajuste al todo social, esto es a las condiciones dadas. La autoactividad valiosa en el niño es aquella que se acompasa con la actividad de las instituciones sociales:

«La regla concreta de la pedagogía es no perder de vista los dos lados, y animar al niño a la autoactividad solo 'en la medida' en que la misma sea racional, es decir, en la medida en que su autoactividad le permita reforzarse a sí mismo con la autoactividad del todo social, o, por decirlo de otra manera, esa regla impone la prescripción sobre el niño solo en la medida en que la misma sea saludable para el desarrollo de su autoactividad» (ibíd.: 815).

Como segunda consecuencia de su concepto hegeliano de educación, para Harris resulta insuficiente todo estudio del niño/a que atienda solo a su ser natural. En el credo Harris critica el movimiento de child study, «que todavía no 
conoce el alfabeto de la psicología racional» (ibíd.). Harris no menosprecia las aportaciones de la psicología empírica en lo que tiene que ver con el estudio de los efectos de los métodos instructivos sobre el crecimiento del niño/a, pero considera que esta queda insuficiente sin una psicología racional que investigue los presupuestos filosóficos de la vida mental y revele el sentido del desarrollo humano.

Es la psicología racional y no la empírica la que proporciona la pauta a que debe atenerse la educación. Harris concreta esta pauta en tres estadios del pensamiento. En el primer estadio, sensorial-perceptivo, los objetos se consideran de manera atomística, sin relación los unos con los otros. En el segundo estadio, de pensamiento panteísta, cada cosa no puede existir al margen de su relación con el todo. En el tercer estadio, se capta que el ser no es ni independiente ni relativo, sino autoactivo y autodeterminado. Este tercer estadio pone en contacto con la necesidad de una razón divina, un absoluto:

«El tercer estadio de pensamiento llega a la intuición de que el verdadero ser es autoactivo o autodeterminado. Es, en consecuencia, ser autoconsciente, y está por encima del intelecto y la voluntad. En tanto que el intelecto es en su naturaleza esencial, altruista, o lo que hace de sí mismo su propio objeto y da existencia objetiva a otros, se desprende que su visión del mundo necesita presuponer una razón divina como el absoluto que crea con el objetivo de poder compartir su ser con otros en su propia imagen» (ibíd.: 814).

Las tres dimensiones del desarrollo del yo y la mismidad se interrelacionan teniendo como referente exterior la razón divina, lo absoluto. Hay una preocupación por el yo reflexivo que se conjuga con lo absoluto. El trabajo de la escuela es hacer avanzar el pensamiento hacia este tercer estadio, que tiene su correlato en el mundo de la voluntad moral. Así como el desarrollo del pensamiento se alcanza mediante la instrucción intelectual, la voluntad moral se logra mediante la disciplina adoptada en la escuela, la cual debe ejercerse ya desde el kindergarten, desarrollando en el niño el poder del autocontrol y la cooperación en vista a fines racionales. En la educación elemental, la disciplina construye el sentido de la responsabilidad. Se trata de adquirir el dominio de sí mismo, resistir las inclinaciones y la fuerza de los hábitos, lo cual es la demostración de la absoluta libertad.

El credo de Harris plasma la confianza en la escuela, como instrumento que hace que todos puedan participar en la vida civilizada, y en la educación y sus métodos para desarrollar tanto la inteligencia como el poder de la voluntad. Los nuevos métodos pedagógicos son, por ejemplo, los «más adecuados para ayudar a los niños cuyas almas están aprisionadas en sus cuerpos y que son aburridos y estúpidos» (ibíd.: 815). Lo son también para hacer subordinar las tendencias egoístas al interés del conjunto, un poder en el que, para Harris, el niño americano destaca sobre el de otros continentes. Pero no todo es bueno en la nueva pedagogía y Harris deplora la visión de algunos kindergarten en los que dos o tres niños/as brillantes monopolizan la atención, no solo de todos los demás compañeros, sino también del maestro/a. «Tales jardines de niños/as nos recuerdan los jardines de cocina que están ahogados con hierbas salvajes» (ibíd.).

Al mismo tiempo que publicaba su credo, Harris reunía gran parte de su pensamiento pedagógico en la obra Psychologic Foundations of Education: an Attempt to Show the Genesis of the Higher Faculties of the Mind, publicada en 1898 como volumen treinta y siete de la colección International Education Series, dirigida por el propio Harris. La introducción contiene los mismos párrafos del credo correspondientes a las limitaciones del child study y las tres fases del pensamiento de la psicología racional, que constituyen motivos centrales del libro.

En esta obra, coetánea del credo, Harris desarrolla una visión del juego paralela a su concepción 
El educando como sujeto y el lugar del juego en el debate educativo de finales del siglo XIX en Norteamérica

del sujeto. El juego es una de las actividades simbólicas que distinguen al ser humano del animal, e incluso cuando se considera una actividad autogratificante, tiene un carácter mediador para insertar al sujeto en el orden social en el que alcanza su ser superior. El juego es un mecanismo de «la recuperación de nuestro sentido de la libertad, en este tránsito del impulso a la obediencia al orden social» (Harris, 1898: 282).

Harris aconseja que «el recreo escolar debiera ser una actividad al aire libre y dedicarse al juego espontáneo, para que la voluntad descanse de su tensión» (ibíd.: 288). El recreo cumple en las escuelas el mismo papel que tienen en las universidades los ritos de iniciación. En un caso y en otro, estas actividades facilitan lo que, basándose en Hegel y Rosenkranz, Harris denomina el proceso de autoextrañamiento o autoalienación (self-estrangement) en que consiste la educación, el paso de un entorno cotidiano a un mundo distinto por descubrir. En estas actividades lúdicas se mantiene el reconocimiento del orden social y la autoridad como algo sustancial, cuya ausencia las convierte en crímenes y deshumaniza la vida, que «no merece la pena ser vivida por el hombre (sic) a menos que pueda participar en la vida de la raza, y así tomar parte de la infinitud» (ibíd.: 289).

Harris tiene muy en cuenta la aportación de Froebel, cuya filosofía del juego considera su principal contribución al kindergarten. Los juegos ofrecen a los niños y las niñas una expresión simbólica de la experiencia acumulada por la humanidad, su sabiduría, y constituyen su primera fuente de autorevelación. El juego tiene de este modo un significado de desarrollo humano, no solo en sentido psicológico, sino fundamentalmente moral. Mediante los juegos regulados, en los que participa con sus compañeros y adultos, el niño/a asciende del mundo de la naturaleza al de la humanidad, del terreno de las cosas al de la autoactividad, de lo material a lo espiritual, de su yo particular a un todo superior: «En el juego regulado (plays and games) se hace consciente de su yo social, y allí amanece el ideal más alto de un yo que se realiza en las instituciones, frente al yo especial del individuo particular» (ibíd.: 316).

La institucionalización del juego desempeña de este modo un papel similar al que cumple el Estado en la evitación del crimen o la Iglesia en la prevención del pecado, «actitudes del individuo que pone su yo inferior en oposición a su yo superior» (ibíd.: 290). La concepción de Harris responde a una visión dualista del ser humano, compuesto de un self inferior, «el cuerpo en que vive y debe utilizar como instrumento con el que actuar» (ibíd.: 292), que puede albergar pasiones y tendencias al pecado, sin por ello anular su libertad, y el self superior que se materializa en las instituciones. El juego institucionalizado o regulado cumple un papel de mediación entre uno y otro, como mecanismo que facilita realizar libremente y sin contradicción la «renuncia a la libertad del momento en pos de la libertad que tiene la forma de la eternidad» (ibíd.: 282).

El biógrafo de Harris, Kurt F. Leidecker, destacó la significación de Psychologic Foundations of Education, que amplía la brecha entre empiristas e idealistas, en la historia intelectual de Estados Unidos (Leidecker, 1946: 535). Pero como toda transición entre una época y otra, la obra culminante de Harris representa también su ocaso. Escribe Leidecker:

«El libro selló su destino como un pensador de la educación. Por paradójico que pueda parecer, al publicar la suma y sustancia de su sabiduría educativa, que él había dado con tanta libertad y que le había reportado tanto éxito personal, Harris se encaminaba al olvido, porque la suya ya no era la voz de la tendencia de los tiempos. La crítica era amable, indulgente, porque Harris había sido amable e indulgente, y su elevado conocimiento no podía ser atacado. Incluso John Dewey, su gran amigo, no podía dejar de colocarlo públicamente en el lado del conservadurismo, sí, incluso reaccionario. Si Harris hubiese publicado el libro veinte años antes, el juicio 
Rosa Bruno-Jofré y Gonzalo Jover

posterior, como juicio de su época, podría haber sido diferente. Pero apareció muy tarde en la historia de la educación norteamericana» (ibíd.: 533-534).

Un juicio similar cabría, por tanto, hacer de My Pedagogical Creed de Harris, que coincide con los motivos centrales de Psychologic Foundations of Education. El credo deja entrever la fuerza con la que se abrían paso otras opciones acerca de la consideración del niño/a como sujeto de la educación y su estudio.

\section{El froebelianismo de James L. Hughes}

El credo de James L. Hughes y sus concepciones del educando y del juego requieren ser situados en relación con su práctica en el contexto escolar de Ontario (Canadá) y su profunda vinculación con las organizaciones educativas americanas y los líderes de la época, como Harris.

Hughes ocupó el puesto de inspector de escuelas en Toronto, en la provincia de Ontario, donde la escolaridad ya era obligatoria y el proceso de urbanización se sentía en la escuela. Más tarde fue inspector jefe, hasta su jubilación en 1913. Formado en la Escuela Normal, fue un reconocido impulsor del kindergarten froebeliano y del trabajo manual. Escribió intensamente, incluyendo el libro Froebel's Educational Laws for All Teachers (Hughes, 1897). Mantuvo una relación dinámica con pedagogos de Estados Unidos, tuvo una presencia fuerte en la National Educational Association (USA) y, por supuesto, de la Ontario Educational Association (Canadá). Fue también buen conocedor del movimiento froebeliano inglés en favor de la educación infantil, que para él tenía en Dickens su principal proponente ${ }^{6}$.

Hughes alterna en su credo indistintamente el término mismidad (selfhood) con el de individualidad, lo cual lo acerca a los pedagogos con influencia hegeliana ${ }^{7}$. Pero no es la intención aquí argumentar su familiaridad con Hegel, de la cual no hay testimonios. Estaba, sí, familiarizado con William T. Harris. El espíritu religioso de Hughes (era miembro activo de la Iglesia Metodista y masón) le lleva a afirmar en el credo que la mismidad del niño/a es el elemento divino en él, declaración que encaja con su primer artículo de fe de que Dios es el Creador, la fuente de vida, la esencia de vida que da el poder de evolución a una vida más alta, y el centro de la unidad universal. El educando tiene inherentemente una naturaleza divina y única a cada individuo. Por eso una de las funciones primordiales de la educación, dice el credo, es «ayudar al desarrollo completo de la individualidad como la base verdadera de la comunidad humana y la unidad de la humanidad con Dios» (Hughes, 1896: 317). El concepto de unidad era muy froebeliano y también protestante. La noción de individualidad/mismidad del niño/a y educando/a conjuga su esencia única con la comunidad. Tiene una dimensión corporal/espiritual y una dimensión relacional social y reflexiva, lo que está de acuerdo con las corrientes teológicas reconstruccionistas de la época. Consecuentemente, el interés del niño/a, sus inclinaciones, y no de la maestra, están en el corazón del proceso de aprendizaje; de una manera que podría decirse herbatiana, la maestra, señala el credo, provee las condiciones de interés adaptadas al estadio de desarrollo del niño/a. Es interesante comprobar cómo la capacidad reflexiva del niño/a, a través de su autoactividad, lo lleva a su desarrollo, que tiene un carácter inmanente, dada su esencia divina, y cómo la educación tiene que estar en armonía con el proceso de evolución universal. En la lectura ecléctica que hace Hughes de Harris, de Froebel y de las influencias, a menudo, vicarias del neohegelianismo, el individualismo no se pierde, aunque, como sucedía en la teología progresista emergente, buscaba un individuo socializado.

El niño/a (educando/a) se desarrolla mentalmente y como persona a través de la autoactividad y la autodirección, que normalmente son consideradas componentes de una dimensión 
reflexiva del yo. La autoactividad, o actividad del yo (activity of selfhood) es central en el credo de Hughes. También se puede relacionar obviamente con Froebel, muy influyente todavía. En el caso de Hughes, como en Froebel, esta noción del desarrollo está ligada a la libertad personal, la autoexpresión, y a la actividad que viene motivada por sí mismo. Pero la actividad del yo es el único camino para que la mente se active y muestre agresivamente su capacidad de apercepción. Otro principio del credo enfatiza la relación con el mundo natural y con el ambiente social, y sitúa al educando como descubridor de problemas y no solo como alguien capaz de resolverlos. Este principio está en relación con otro que afirma que el niño/a no solo adquiere conocimiento sino que al mismo tiempo debe usarlo tanto como sea posible. Estos son elementos importantes en el marco de las necesidades que afloran del contexto socioeconómico e intelectual, en particular, la emergencia del método científico.

El credo enfatiza la disciplina, que no confunde con control, y relaciona con el concepto de libertad. Así dice que «la ley perfecta de la libertad» hace que la disciplina sea un proceso para la evolución de la divinidad del niño/a. Pero ¿cómo entiende esa libertad? En el libro que Hughes dedicó a Froebel plasma su concepción de la misma en relación con el juego. Por un lado, la autoactividad, motivada por el interés, actuaría como proceso intermediario creando armonía entre espontaneidad y control, cuyo exceso crearía un yo y una mismidad pasiva (Hughes, 1897: 23). La autoactividad es un principio vital y el juego que, siguiendo a Froebel, tiene en Hughes un lugar central en el trabajo escolar, es entendido como un medio para crear la armonía mencionada. No hay que olvidar que el juego era también entendido en Alemania como un factor importante en el desarrollo físico de la raza, punto que Hughes reconoce (ibíd.). El juego regulado u organizado es parte de la idea de que es imposible concebir la libertad sin una conciencia de lo que significa la restricción (ibíd.: 83). Escribe:
«La ley de la reconciliación de contrarios u opuestos debiera permitir a los maestros entender la armonía entre control y espontaneidad. Deberían hacer de esto las bases de la ley de la disciplina. Es la 'Ley perfecta de la libertad'. La espontaneidad perfecta y la sumisión completa a la ley son amantes cercanos. Todo maestro debería consagrar la unión de estos amantes en la vida del niño. Ningún maestro está totalmente cualificado hasta que esté preparado para este alto servicio» (ibíd.).

El juego tenía que ser sistematizado y dirigido con gran delicadeza, idea froebeliana, dado que ayuda a desarrollar las cualidades morales que se conciben como necesarias. Así, Hughes entendió que el juego y el trabajo estaban relacionados. Dentro de la concepción dominante de la masculinidad y la femineidad, Hughes afirma que el juego del niño corresponde al trabajo del hombre. No deja de agregar al pie de página que la niña debiera jugar tanto como el niño, ya que las madres de cada generación deben moverse hacia un cuerpo y un cerebro cada vez más físicamente perfecto (ibíd.: 139).

Hughes en su credo enfoca al educando y su mismidad desde una perspectiva muldimensional. Cabría decir que todos los elementos de la época estaban de alguna manera presentes de una manera bastante ecléctica, pero respondiendo a la realidad. Su comprensión de la dimensión material corporal tiene una base biológica natural y divina y está íntimamente entrelazada con la dimensión relacional y reflexiva. Escribe que la naturaleza física, intelectual y espiritual deben ser entrenadas como una unidad. No descuida el desarrollo del cerebro, la coordinación del sistema nervioso, el cultivo de la mente e incluso menciona la nutrición adecuada del cerebro y los ejercicios físicos.

Su visión del educando es la de un ser libre, capaz de funcionar creativamente en su medio, descubriendo y resolviendo problemas, autoactivo y capaz, consciente de su lugar en la historia de la civilización humana. Sin embargo, 
Hughes deja poco claro el papel de los ejercicios militares y gimnásticos que auspició en su práctica.

En el contexto de la autoactividad y la libertad, que está obviamente condicionada en el contexto escolar, el credo de Hughes y su obra sobre Froebel, esencial para entender el credo, contrasta con el de Scripture, que veremos a continuación, quien buscaba la obediencia como uno de los objetivos en la formación del educando/a. Las ideas de Stanley Hall, quien fue llamado en su tiempo el Darwin de la mente, y el movimiento de child study aparecen reflejados en el credo de Hughes cuando habla del perfil del educando e invoca las épocas culturales, recreación de estadios culturales como un medio de desarrollo del poder individual y del carácter.

\section{El positivismo de Edward W. Scripture}

El credo de Edward Wheeler Scripture representa una nueva lectura frente a los de Harris y Hughes. Veintinueve años más joven que el primero y dieciocho que el segundo, fue uno de los promotores de la «nueva psicología» americana, denominación que él usó para referirse a la psicología experimental que se traía de Alemania y se americanizaba en el proceso de recepción.

En su texto, Scripture pone énfasis en la necesidad de controlar al niño, moldear su mismidad, y expone la necesidad de limitaciones y restricciones dentro de lo que sería el desarrollo de las relaciones interpersonales y con el conjunto de la sociedad. Así, entiende que una obligación primordial de la educación es formar al niño/a en la obediencia y el autocontrol, favoreciendo los ejercicios de tipo militar. Su deseo es que el niño/a llegue a prestar atención y siga el proceso instructivo voluntariamente y alegre. Al mismo tiempo, se preocupa de las condiciones físicas del niño/a, el ambiente (aire y luz), considera las necesidades corporales y, en consecuencia, cuestiona lo que llama el sistema de instrucción que no tiene en cuenta los límites naturales físicos del niño/a, tal como su capacidad de atención, o las necesidades inherentes a su desarrollo.

Los artículos de su credo representan un ataque bastante frontal a los neohegelianos y a los seguidores de Froebel, en especial a los postulados derivados de una lectura hegeliana de este, algo no difícil de hacer. En realidad, Scripture rechaza las bases fundacionales metafísicas a favor de un enfoque empirista que puede construirse como positivista frente a los credos de Harris y Hughes. Scripture «cree» que a los niños/a se les debiera, al menos, permitir amar la belleza. Explica que cuando los niños/as hacen ribetes $u$ otros diseños con papeles coloreados, no se les debiera castigar por usar colores brillantes. Se pregunta si es necesario que los materiales tengan colores no definidos. Concluye este punto diciendo que «esto es el resultado natural de las ideas que vienen de Inglaterra donde se vive en una niebla continua, atmosférica e intelectual. Pero América tiene sol y puede hacer las cosas más placenteras. Dejemos que los niños/as tengan algo de sol en sus colores y no se les diga que la pizarra, el lápiz y la tiza son más bonitos que las cerezas y las ciruelas» (Scripture, 1896: 621). Con ello, hace una clara referencia al neohegelianismo y al énfasis en el conocimiento racional, es decir, a la idea de que la razón tiene más importancia que los sentidos en el proceso de conocimiento. El acento en la experiencia sensorial lo relacionaría con empirismo y, eventualmente, con positivismo y su confianza no solo en la experiencia sensorial sino en la aplicación de los métodos de las ciencias naturales a los estudios sociales. Los niños/as no debieran ser enseñados a amar lo que es feo porque deforma su sentido de la belleza. Cuestiona el uso de tablillas de colores y prescribe el empleo de algo natural y real. En ese sentido, cree que no se debiera permitir a la industria y a la gente de negocios determinar los métodos de instrucción. 
El educando como sujeto y el lugar del juego en el debate educativo de finales del siglo XIX en Norteamérica

Los principios del credo de Scripture muestran su crítica al idealismo, a veces entretejido con nuevas concepciones en psicología y filosofía. Así, establece como principio que la instrucción se base en la «verdad» tanto como sea posible. Da un ejemplo expresivo de su posición. Enseñar en el jardín de infancia que el niño/a es el motivo para que la naturaleza florezca y los seres humanos trabajen, es una mentira medieval. Si el niño/a tiene un espíritu saludable rápidamente rechaza esas estupideces. Enseñar mentiras es, a su juicio, un crimen. Más aún, el niño/a debiera tener alguna idea acerca de lo que se habla, algún punto de contacto real, en vez de imágenes. Esto conduce a lo que se podría decir es el punto central de su credo. Scripture cree:

«Que la instrucción en metafísica mística está fuera de lugar en el kindergarten. Noah Porter — ¿o fue Dr. McCosh?_- dijo alguna vez que las viejas filosofías alemanas se fueron a Oxford y Cambridge cuando ya estaban muertas. La filosofía hegeliana se marchó allí. Esto ocurrió muchos años atrás. Ahora la filosofía hegeliana ha encontrado su hogar en el kindergarten americano. Todo simboliza algo. La esfera simboliza el universo, el sol, la tierra, la luna (¿por qué no la naranja, la uva y la burbuja de jabón?). El cilindro es un símbolo - puede hacerse una lista consultando los libros de kindergarten- Nada es lo que es, y todo es lo que no es. Puede que esto no se entienda, pero recuérdese que el mismo Hegel dijo que 'Solamente un hombre había entendido su filosofía y, después de todo, tampoco la había asimilado realmente'. Dejemos el misticismo, asumamos el sentido común» (ibíd.: 622).

El niño/a como ser biológico ocupa un lugar central en el credo de Scripture, mostrando filosóficamente la influencia darwiniana. Uno de los artículos del credo se refiere al derecho inalienable del niño/a a la salud y a la felicidad y, si está obligado a ir a la escuela, esta tiene que asegurar condiciones saludables, así como darle la oportunidad y el ambiente para que su mente pueda trabajar.
Su método de educación del niño/a consistiría, según el credo, primero en ejercicios físicos, sobre todo en el exterior, trabajo manual, escritura y dibujo, trabajos artísticos. El jardín de infancia o kindergarten debiera ocuparse con juegos regulados (games), en el interior y en el exterior, en vez de dedicarse a hacer trabajos con bolitas y otros elementos, o a la costura, actividades que encuentra abominables. Después del jardín de infancia, la instrucción principal, además de la suma y resta y la lengua, se ocuparía del trabajo de la madera. El trabajo manual tendría que estar siempre incluido en la instrucción. Scripture no se detiene en el análisis del juego espontáneo, imitativo de la vida misma. Su base experimental, expresada en su trabajo académico, lo lleva a la observación y medición de los niños en términos de su constitución física, funcionamiento, y actividades. Los juegos regulados cumplen en este contexto un papel importante en línea con el objetivo de que los niños aprendan autocontrol en el camino de su desarrollo social y personal (Scripture, 1894 y 1895). En los años ochenta y comienzos de los noventa, Scripture llevó a cabo el primer estudio sistemático riguroso sobre la influencia del ejercicio físico en la personalidad, estudiando a jóvenes recluidos en el Elmira Reformatory (Nueva York). Concluyó que había efectos positivos en relación con el autocontrol y la conducta de los jóvenes que participaban en programas de atletismo, gimnasia y trabajo manual. El diseño de estos estudios partía de la premisa de que el ejercicio físico construye el carácter, siendo transferible a la vida diaria (Green y Benjamin, 2009: 101-102).

Podemos concluir que en la visión de Scripture, el educando debiera formarse en relación con el mundo natural y social; el cuerpo, en línea con el movimiento higienista, es importante y la escuela debe respetarlo. Postula una enseñanza basada en la verdad y en los hechos, alejada del misticismo idealista; una enseñanza realista que esté atenta al «lado bueno de la vida», lo que supone apartar al niño/a de lo que él llama monstruosidades en las relaciones 
Rosa Bruno-Jofré y Gonzalo Jover

sociales. Así, se opone a la inclusión de alumnos «con defectos» en su conducta o en su físico (epilépticos, «idiotas»). El niño como individuo tiene un lugar central. Sin embargo, no hay preocupación por el desarrollo reflexivo. La conclusión en su credo sintetiza su posición. «Está llegando el buen tiempo [...] en el que la educación será un arte basado en principios científicos y no en una mezcolanza de filosofías anticuadas, psicologías vagas, innumerables modas y carencia de sentido sin límite» (Scripture, 1896: 623).

\section{Conclusión}

Los tres credos seleccionados ilustran la internacionalidad y el movimiento de las ideas pedagógicas a fines del siglo XIX, así como sus reconfiguraciones a través de lecturas mediadas por el contexto histórico. Los credos elegidos para delinear las nociones del yo, la mismidad, y el educando en relación con concepciones de la buena vida y el lugar del juego en el crecimiento individual y social, muestran posiciones contrastantes con respecto a las dimensiones material, relacional y reflexiva. El racionalismo idealista de Harris, sus estadios de desarrollo del pensamiento y su compleja noción de la autoactividad como puente entre el individuo y la sociedad, llevando al niño/a a formas altas de actividad del alma, contrasta con el empirismo de Scripture. Hughes muestra en su credo una conjugación ecléctica de la pedagogía y filosofía alemana y europea y una fuerte influencia de Froebel. Sus concepciones están mediadas por sus actividades en Estados Unidos y Canadá y su trabajo como inspector en las escuelas de Toronto.

Para Harris, el juego es un mecanismo de integración del individuo en el todo social, un instrumento de desarrollo moral. Mediante el juego regulado, el sujeto renuncia a la libertad individual, para subordinase a la norma y los hábitos sociales, que se identifican con una libertad superior, encarnada en las instituciones. Hughes sigue en parte a Harris, pero sobre todo a Froebel, en la importancia que da al juego y a la autoactividad como un proceso que crea armonía entre espontaneidad y control. El juego regulado aparece ligado a su idea de libertad, la cual no puede existir sin limitaciones. Con respecto a los dos anteriores, Scripture representa un cambio paradigmático, ya que se mueve hacia una visión fuertemente experimental de la educación, que conduce a la estandarización del sujeto. Los juegos regulados son medios de desarrollo psicológico y físico, susceptibles de ser estudiados empíricamente, pero también medios de autocontrol con fines personales y sociales.

En los tres autores estudiados el juego aparece como un medio formativo fundamental en el desarrollo no solo individual, sino también social de los niños/as, lo que hoy llamaríamos una educación para la ciudadanía. El surgimiento de la psicología empírica y el énfasis puesto en la experiencia sensorial llevan al debate sobre el carácter idealista o realista de los instrumentos usados en el juego educativo, los actuales juguetes didácticos. Su inserción en una intencionalidad educativa impone condiciones especiales al juego. Así, se trate de una educación y visión del sujeto de carácter humanístico, como en Harris, froebeliana, como en Hughes, o experimental, como en Scripture, el juego, sea espontáneo o regulado, se convierte en un mecanismo que lleva a la noción del sujeto autocontrolado para su inclusión en el todo social, dejando de ser estrictamente una actividad lúdica. 
El educando como sujeto y el lugar del juego en el debate educativo de finales del siglo XIX en Norteamérica

\section{Notas}

${ }^{1}$ Un artículo muy valioso para una lectura contextualizada de la influencia neohumanista alemana, es el de Johannes Bellmann, «Reinterpretation in Historiography: John Dewey and the Neo-Humanist Tradition». El autor cuestiona la tesis de Jürgen Oelkers, quien subraya la discontinuidad que supone Dewey con la tradición de la filosofía de la educación alemana. En vez de ello, Bellmann ubica continuidades aun donde Dewey trata de mostrar ruptura. El artículo discute procesos de recepción y readaptación de Hegel y Herbart en el pensamiento de Dewey (Bellmann, 2004).

2 Véase, por ejemplo, Hofstetter y Schneuwly (2004) y Fuchs, Lindmark y Lüth (2007).

${ }^{3}$ Puede verse al respecto Carter (1966).

${ }^{4}$ Utilizamos la palabra alemana kindergarten, que era la habitual en los credos.

${ }_{5}^{5}$ En este libro se separa de lo que entiende puede ser una derivación panteísta de la filosofía hegeliana, si bien, a su juicio, esta dificultad «no contamina la teoría hegeliana de la vida humana y de la iglesia cristiana» (Harris, 1890: XIV).

${ }^{6}$ Véase su libro Dickens as an educator, que hace el volumen cuarenta y nueve de la International Education Series, dirigida por Harris (Hughes, 1901).

${ }^{7}$ Das Selbst es una categoría central en la filosofía de Hegel, en quien, como indica Seigel, «el isomorfismo de sí mismo (self) y mundo, proyectado por sus predecesores alemanes, alcanza su forma más elaborada y consecuente» (Seigel, 2005: 391).

\section{Referencias bibliográficas}

Bellmann, J. (2004). Reinterpretation in Historiography: John Dewey and the Neo-Humanist Tradition, Studies in Philosophy and Education, 23 (5), 467-488.

Bruno-Jofré R. y Jover, G. (2009). El ideal democrático en el ideario pedagógico americano de finales del siglo XIX y su transposición en dos escenarios de habla hispana. En: M. R. BerRUEzO y S. Conejero (eds.), El largo camino hacia una educación inclusiva. Pamplona: UPN, 23-33.

Bruno-Jofré R. y Jover, G. (2011). The Pedagogical Creeds of the End of the XIX Century in the United States, the Transatlantic Movement of Ideas, and the Readings of John Dewey's Creed in its Intersection with Catholicism. Ponencia presentada en Canadian Catholic Historical Association / American Catholic Historical Association Joint Conference. Toronto, St. Michael's College / University of Toronto, 15-16 de abril de 2011.

Carter, B. N (1966). James L. Hughes and the Gospel of Education: A Study of the Work and Thought of a Nineteenth Century Canadian Education. Thesis submitted for the Degree of Doctor of Education, University of Toronto.

Cremin, L. A. (1964). The Transformation of the School: Progressivism in American Education, 18761957. New York: Alfred A. Knopf.

Fuchs, E.; Lindmark, D. y LüTh, C. (eds.) (2007). Networks and the History of Education, Paedagogica Historica, 43 (2), 185-197.

Green, C. D. y Benjamin, L.T. (eds.) (2009). Psychology Gets in the Game: Sport, Mind, and Behavior, 1880-1960. Lincoln: University of Nebraska Press.

HARris, W. T. (1890). Hegel's logic. A book on the genesis of the categories of the mind. A critical exposition. Chicago: S. C. Griggs \& Co.

Harris, W. T. (1897). My Pedagogical Creed. The School Journal, LIV (26), 813-815.

HARris, W. T. (1898). Psychologic foundations of education: an attempt to show the genesis of the higher faculties of the mind. New York: D. Appleton \& Co. 
Rosa Bruno-Jofré y Gonzalo Jover

Hofstetter, R. y Schneuwly, B. (eds.) (2004). The Role of Congresses and Institutes in the Emergence of the Educational Sciences, Paedagogica Historica, special issue, 40 (5-6).

Hughes, J. L. (1897). Froebel's educational laws for all teachers. New York: D. Appleton and Co.

Hughes, J. L. (1901). Dickens as an educator. New York: D. Appleton and Co.

Hughes, J. L. (1896). My Pedagogical Creed, The School Journal, LIII (12), 317-18.

Jenkins, P. (2010). Breve historia de los Estados Unidos. Madrid: Alianza.

Kliebard, H. M. (1986). The Struggle for the American Curriculum, 1893-1958. New York: Routledge \& Kegan Paul.

Lang, O. H. (1898). Educational Creeds of the Nineteenth Century. New York and Chicago: E. I. Kellogg \& Co. (reeditado por Kessinger Publishing's Rare Reprints).

LeIDECKer, K. F. (1946). Yankee Teacher. The Life of William Torrey Harris. New York: The Philosophical Library.

SCripture, E. W. (1894). Aims and Status of Child Study, Educational Review, 8 (3), 236-239.

Scripture, E. W. (1895). Child Study: Methods and Results. En Sixty-Fifth Annual Meeting of the American Institute of Instruction: Lectures, Discussions, and Proceedings. Boston: American Institute of Instruction, 181-188.

SCRIPTURE, E. W. (1896). My Pedagogic Creed, The School Journal, LIII (21), 621-623.

SEIGEL, J. (2005). The Idea of the Self: Thought and Experience in Western Europe Since the Seventeenth Century. Cambridge: Cambridge University Press.

The School Journal (1896). Note to My Educational Creed, by Heber Holbrook, The School Journal, LIII (22), 662.

TrÖHLER, D. (2011). The Becoming of an Educational Science: Protestant Souls and Psychologies. En Languages of Education, Protestant Legacies, National Identities, and Global Aspirations. New York: Routledge, 131-147.

\section{Abstract \\ The learner as subject and the place of play and games in the educational debate in North-America at the end of 19th century}

The article examines the building of the idea of the learner as subject and related notions of play and games, in three of the pedagogical creeds published in The School Journal (New York and Chicago) between 1896 and 1897. The creeds are those of American William Torrey Harris (18351909), a Hegelian humanist, Commissioner of the USA Bureau of Education; James L. Hughes (1846-1935), Canadian school inspector from Toronto, an advocate of Froebel's ideas; and Edward W. Scripture (1864-1945), a professor of Psychology at Yale University, specialist in experimental psychology. Jerrold Seigel's differentiation of the three dimensions of self-hood, material or corporal, relational, and reflective, provide the theoretical framework.

We argue that in Harris's vision of the learner the relational dimension is dominant and aims at an integration of the individual in the social whole. Hughes conceives self-activity as a mediating element between control and spontaneity, having as frame of reference a notion of freedom that cannot be understood without a consciousness of what restriction meant. Scripture embodies the paradigmatic change taking place. His vision of the self and the learner as subject is influenced by the natural sciences and the emerging empiricist methodology, in line with positivist thinking. The three authors understand play and games as means to develop a self-controlled subject responding to an external intentionality even as the debate takes the shape of idealism versus realism (the 
El educando como sujeto y el lugar del juego en el debate educativo de finales del siglo XIX en Norteamérica

latter expounded by Scripture). In the end, play and not only games lose their meaning as strictly ludic activities.

Key words: Nineteenth century education, Learner, Subject, Self, Play, Games.

\section{Résumé}

L'apprenant comme sujet et le lieu du jeu et des jeux dans le débat éducatif en Amérique du Nord à la fin du 19e siècle

L'article examine le développement de l'idée de l'apprenant comme sujet et les notions de jeu et de jeux, dans trois des crédos pédagogiques publiés dans The School Journal (New York et Chicago) entre 1896 et 1897. Les crédos sont ceux de l'Américain William Torrey Harris (1835-1909) un humaniste Hégélien, commissaire du Bureau d'éducation des États-Unis; James L. Hughes (18461935), inspecteur canadien des écoles de Toronto, un défenseur des idées de Froebel; et Edward W. Scripture (1864-1945), professeur de Psychologie à l'Université Yale, spécialiste en psychologie expérimentale. La différenciation des trois dimensions du soi, matérielle ou corporelle, relationnelle et réflexive, fournit le cadre théorique.

Nous soutenons que dans la vision de l'apprenant de Harris la dimension relationnelle est dominante et vise à l'intégration de l'individu dans le tout social. Hughes conçoit l'autoactivité comme élément médiateur entre le contrôle et la spontanéité, ayant comme cadre de référence une notion de la liberté qui ne peut pas être comprise sans une conscience de ce que veut dire la restriction. Scripture exprime le changement paradigmatique qui s'opère. Sa vision du soi et de l'apprenant comme sujet est influencée par les sciences naturelles et la méthodologie empiriste naissante, en ligne avec la pensée positiviste. Les trois auteurs comprennent le jeu et les jeux comme moyens de développer un sujet autodiscipliné répondant à une intentionnalité externe au moment même où le débat prend la forme de l'idéalisme versus le réalisme (ce dernier expliqué par Scripture). À la fin, le jeu et non seulement les jeux perdent leur signification comme activités strictement ludiques.

Mots clés: Éducation au dix-neuvième siècle, Apprenant, Sujet, Soi, Jeu, Jeux.

\section{Perfil profesional de los autores}

\section{Rosa Bruno-Jofré}

Catedrática y anterior decana (2000-2010) de la Facultad de Educación de Queen's University (Canadá). Directora del Theory and History of Education International Research Group. Ha publicado numerosos artículos y varios libros, entre otros: The Missionary Oblate Sisters: Vision and Mission (McGill-Queen's University Press, 2005; edición en francés de 2008) y Democracy and the Intersection of Religion and Traditions (McGill-Queen's University Press, 2010) con J.S. Johnston, G. Jover y D. Tröhler.

Correo electrónico de contacto: brunojor@queensu.ca. 


\section{Gonzalo Jover}

Catedrático de Teoría de la Educación en la Universidad Complutense de Madrid. Asesor del Vicerrectorado de Posgrado y Formación Continua y coordinador del Máster en Formación del Profesorado de Educación Secundaria. Dirige el Grupo de Investigación sobre Cultura Cívica y Políticas Educativas. Ha publicado numerosos artículos y varios libros, entre otros: Ética profesional docente (Síntesis, 2010), con J. Escámez y R. García López, y Democracy and the Intersection of Religion and Traditions (McGill-Queen's University Press, 2010), con R. Bruno-Jofré, J. S. Johnston y D. Tröhler. Promotor y Director del Observatorio del Juego Infantil.

Correo electrónico de contacto: gjover@edu.ucm.es 